\title{
Advances in Cancer Therapy: Immunotherapies
}

\section{Dieter Marmé}

Klinik für Tumorbiologie, Freiburg i.Br., Germany

For a long time, progress in cancer therapy only occurred in small steps. However, with the increasing knowledge of the mechanisms and molecules involved in tumor development, this has changed considerably over the past 20 years.

The early 90's saw the successful development of targeted therapies. Targets involved in the progression of tumors were identified and validated, and drugs that were shown to interfere with the activity of such targets were developed and confirmed to be clinically effective. Examples are HER2 receptors / trastuzumab, BCL-Abltyrosine kinase / imatinib, and VEGF / bevacizumab. Numerous drugs of this type, inhibiting either just one target or more, have now made their way into the clinics. Monotherapies and/or combinations have been shown to be more successful when used in the context of appropriate diagnostic procedures.

Recently, a paradigmatic change in cancer therapy occurred with the development of checkpoint inhibitors. These molecules, mostly antibodies, interfere with the immune response against tumors. The first compound of this type, ipilimumab, which was approved in 2011 for the treatment of malignant melanoma, is a monoclonal, human antibody against the T-cell checkpoint molecule CTLA-4. A second antibody, nivolumab, directed against the receptor programmed cell death protein 1 (PD-1), seems to be even more effective in the treatment of melanoma, renal cancer, and lung cancer. The mode of action of these checkpoint inhibitors is basically to counteract the inhibitory function of specific $\mathrm{T}$ cells and, as a consequence, activate their potential to eliminate the recognized tumor cells. The advantage of this type of immunotherapy appears to be that, once the immune reaction has been activated, these $\mathrm{T}$ cells can also address new targets on tumor cells that might show up over time and that, due to their circulation throughout the whole body, they can target and eliminate tumor cells wherever they hide on their metastatic route.

Quite a number of new agonistic as well as antagonistic antibodies are being developed clinically to improve the therapeutic outcome in various types of tumors. In particular, by applying those new molecules, it is hoped to address the tumors of those patients who show only weak responses or no response at all. This also implies the need for focused basic research to gain a better understanding of the interaction of tumor cells and $\mathrm{T}$ cells and, as a consequence, the functional outcome of these interactions. Thus, it will be of interest to see whether the blockade of other co-inhibitory T-cell antigens such as LAG-3 or the activation of co-stimulatory T-cell antigens such as CD137 can contribute to the elimination of tumor cells. On a laboratory scale, interesting co-culture systems using patients' tumor cells and $\mathrm{T}$ cells have been developed to investigate these open questions.

Furthermore, clinical trials are being carried out to explore whether combinations of 2 checkpoint inhibitors have an advantage over the respective monotherapies. It will also be of interest to see if there is a therapeutic benefit when checkpoint inhibitors are combined with other targeted strategies such as inhibitors of tumor angiogenesis.

The use of checkpoint inhibitors in cancer therapy has some considerable implications as far as toxic side effects are concerned. The cellular and molecular mechanisms of action of these novel drugs imply that they cause immune-related adverse events (irAE). These include tissue destruction and autoimmunity. Therefore, a continuous and careful monitoring is essential to apply the appropriate treatment algorithms.

These advances in cancer immunotherapy are very promising not only because they provide more efficient treatment for some patients, but even more because they provide a concept for a paradigmatic change in cancer therapy. Very recently, Gros and colleagues [1] successfully isolated tumor-reactive $\mathrm{CD}^{+} \mathrm{T}$ cells expressing PD-1 from the circulating blood of patients with melanoma. This, as Schumacher and Scheper [2] put it in their 'News and Views' commentary, provides a possibility for simply isolating and propagating tumor-reactive, $\mathrm{PD}-1$ expressing T cells that could be reinfused into the patient and would recognize and bind to the patients 'private' neoantigen(s). Subsequent treatment with PD1 -specific antibodies such as nivolumab could booster the T-cell response and enhance tumor elimination.

The following Minireviews summarize our current knowledge about the clinical benefit of immunotherapies in brain [3], breast [4], colorectal [5], lung [6], kidney [7], and skin [8] cancer.

\section{KARGER \\ Fax +497614520714

(c) 2016 S. Karger GmbH, Freiburg

$2296-5270 / 16 / 0396-0324 \$ 39.50 / 0$
Prof. Dr. Dieter Marmé 


\section{Disclosure Statement}

D.M. does not receive any funding and/or honoraria from pharmaceutical companies.

\section{References}

1 Gros A, Parkhurst MR, Tran E, et al.: Prospective identification of neoantigen-specific lymphocytes in the peripheral blood of melanoma patients. Nat Med 2016;22:433-438.

2 Schumacher TN, Scheper W: A liquid biopsy for cancer immunotherapy. Nat Med 2016;22:340-341.

$\checkmark 3$ Roth P, Preusser M, Weller M: Immunotherapy of brain cancer. Oncol Res Treat 2016:DOI: 10.1159/ 000446338 .
4 Marmé F: Immunotherapy in breast cancer. Oncol Res Treat 2016:DOI: 10.1159/000446340.

5 Jäger D, Halama N, Zörnig I, Klug P, Krauss J, Haag G: Immunotherapy of colorectal cancer. Oncol Res Treat 2016:DOI: 10.1159/000446713.

6 Reinmuth N, Reck M: Immunotherapy of lung cancer. Oncol Res Treat 2016:DOI: 10.1159/000446726.
7 Grünwald V: Checkpoint blockade - a new treatment paradigm in renal cell carcinoma. Oncol Res Treat 2016:DOI: $10.1159 / 000446718$

8 Bender C, Hassel J, Enk A: Immunotherapy of melanoma. Oncol Res Treat 2016:DOI: 10.1159/000446716. 